\title{
Enhancing the Butyrylcholinesterase Activity in HEK-293 Cell Line by Dual-Promoter Vector Decorated on Lipofectamine
}

This article was published in the following Dove Press journal:

Drug Design, Development and Therapy

\author{
Vida Mirzaie (1D \\ Touba Eslaminejad ${ }^{2}$ \\ Homayoon Babaei ${ }^{3}$ \\ Seyed Noureddin \\ Nematollahi-Mahani ${ }^{4,5}$ \\ 'Department of Anatomy, Afzalipour \\ School of Medicine, Kerman University of \\ Medical Sciences, Kerman, Iran; \\ ${ }^{2}$ Pharmaceutics Research Centre, \\ Institute of Neuropharmacology, Kerman \\ University of Medical Sciences, Kerman, \\ Iran; ${ }^{3}$ Department of Clinical Sciences, \\ Faculty of Veterinary Medicine, Shahid \\ Bahonar University of Kerman, Kerman, \\ Iran; ${ }^{4}$ Neuroscience Research Centre, \\ Institute of Neuropharmacology, Kerman \\ University of Medical Sciences, Kerman, \\ Iran; ${ }^{5}$ Afzal Research Institute (NGO), \\ Kerman University of Medical Sciences, \\ Kerman, Iran
}

Purpose: Human butyrylcholinesterase (BChE) serves as a bio scavenger to counteract organophosphate poisoning. It is also a potential drug candidate in several therapeutic fields. Therefore, in the present study, we constructed a new dual-promoter plasmid consisting of Cytomegalovirus (CMV) and human elongation factor $1 \alpha(\mathrm{EF}-1 \alpha)$ promoters and transfected that into HEK-293 cells using Lipofectamine to enhance the BChE secretion.

Methods: The new dual-promoter construction (pBudCE dual BChE) including two copies of the BChE gene was designed and transfected into cells by liposomal structures. The cloned plasmids were evaluated by enzyme digestion and gel electrophoresis analysis. Experimental groups were categorized into the cells transfected by pBudCE dual BChE (treatment), pCMV (positive control) vectors, and nontransfected cells (negative control). $B C h E$ gene expression was evaluated by qRT-PCR and the enzyme activity was assessed using modified Ellman's method. The freeze-thaw process was carried out for analyzing the stability of the pBudCE dual BChE vector.

Results: Validation examination of the cloned plasmids confirmed the successful cloning process. The gene expression level and Ellman's method value in pBudCE dual BChE was higher than the other groups. CMV promoter has also increased the enzyme activity, although the difference was not significant compared with the control group. Interestingly, freeze-thaw cycles followed by several passages did not affect the enzyme activity.

Conclusion: The designed construction with CMV and EF- $1 \alpha$ promoters could increase BChE gene expression and the activity of the BChE enzyme in HEK-293 cell line. Largescale production of $\mathrm{BChE}$ enzyme can be achieved by using dual-promoter plasmid construction compared to a single-promoter vector to be used in clinical trials.

Keywords: dual-promoter, vector, recombinant protein, drug delivery, Ellman's method, HEK-293

\section{Introduction}

Cholinesterases are a range of circulating enzymes present in tissues or in body fluids and plasma. They are divided into two main groups of acetylcholinesterase and butyrylcholinesterase $(\mathrm{BChE})$ based on their behavior in the presence of specific substrates and susceptibility to the limiting agents. ${ }^{1}$ Human BChE is a tetrameric blood globulin glycoprotein consisting of 4 subunits that are stabilized with PRAD peptide and each subunit contains 574 amino acids and 9 carbohydrate chains. $^{2,3}$ The tetramer form of BChE has a long half-life, while monomer and dimer forms will remove from the blood after a few minutes and have less
Correspondence: Seyed Noureddin

Nematollahi-Mahani

Tel +983433257343

Email nnematollahi@kmu.ac.ir 
pharmacological effect. ${ }^{4}$ Serum $\mathrm{BChE}$ is pharmacologically and toxicologically important, since the enzyme has the ability to hydrolyze ester drugs such as succinylcholine and cocaine and converts bambuterol into bronchodilating drug terbutaline. ${ }^{5} \mathrm{BChE}$ can be used either prophylactically or as a treatment drug. One of the most important applications of $\mathrm{BChE}$ is detoxifying organophosphate toxins. Organophosphates or carbamate esters are used in insecticide, pesticides, chemical weapons of mass destruction, and some medicines to treat diseases such as glaucoma, Alzheimer's, and parasitic infections., ${ }^{1,6}$ According to the World Health Organization (WHO), 200,000 people die annually due to poisoning from organophosphate pesticides in developing countries. ${ }^{7-9} \mathrm{BChE}$ is the only bio scavenger known as a "new development drug" in the FDA in 2006 which can prevent poisoning from organophosphate toxins. ${ }^{10,11}$ The use of external BChE $200 \mathrm{mg}$ per $70 \mathrm{~kg}$ body weight has been effective in preventing acetylcholinesterase inactivation. ${ }^{12}$ However, BChE could be isolated from the blood plasma, and several investigations have been done in the past 20 years in order to produce higher amounts of the enzyme. According to studies, a high volume of human plasma (100 liters) is required to produce $200 \mathrm{mg}$ (one dose) of the BChE enzyme, which is expensive, and has the potential of contamination. ${ }^{13,14}$ As an alternative method, it is possible to produce $\mathrm{BChE}$ as a recombinant protein in prokaryotic or eukaryotic cells. ${ }^{15,16}$ The expression of protein in prokaryotic cells is relatively simple and economically affordable. However, scientists could not successfully produce active $\mathrm{BChE}$ in Escherichia coli bacteria. ${ }^{17} \mathrm{~A}$ recent study has shown successful recombination of $\mathrm{BChE}$ in bacteria, although the activity of the produced enzyme for detoxification remains to be evaluated. ${ }^{18}$ The expression of recombinant proteins requires the use of special expression vectors, promoters, and cell lines. ${ }^{19}$ As an appropriate cell line, HEK-293 has been used extensively as a standard host for the cloning process since $1974 .^{20}$ Cytomegalovirus immediate-early promoter (CMV) and human elongation factor $1 \alpha$ (EF$1 \alpha)$ are two of the strongest promoters in gene expression. Jane et al showed that EF-1 $\alpha$ could be strong in all types of cells consistently, while CMV is slightly weaker than EF- $1 \alpha$, and also has variable effects that are very strong in some cell types and rather weak in others. ${ }^{21}$ Although many studies worked on the enhancement of $\mathrm{BChE}$ production using different methods, introducing two copies of the BChE gene in front of the two mentioned promoters, simultaneously in one plasmid, with the aim of increasing the enzyme production has not been studied yet. The present study investigated the effect of the simultaneous use of two promoters of CMV and EF- $1 \alpha$ in a single plasmid on the $B C h E$ gene expression level and enzyme activity in the HEK-293 cell line. The transient stability of the new construction in HEK-293 cells was also evaluated in several freeze-thaw cycles.

\section{Materials and Methods Materials}

The research was approved by the ethical committee of Kerman University of Medical Sciences and the Ethics Approval Code is IR.KMU.REC.1398.294. Restriction enzymes, T4 DNA ligase, GeneJET Plasmid Miniprep ${ }^{\circledR}$ kit (\#K0502, \#K0503), and Taq DNA Polymerase Master Mix RED were purchased from Thermo Scientific (Germany) and Ampliqon ${ }^{\circledR}$ (Denmark) respectively. Plasmids pCMV3-BChE (Cat No., HG12010-UT) and pBudCE4.1 (Cat No., V532-20) were provided by Sino Biological (China) and Invitrogen (USA). RiboEx ${ }^{\mathrm{TM}}$ (Cat No., 301-001) was purchased from GeneAll (Portugal). Parstous cDNA synthesis kit (Cat No., A101161) was provided by Parstous Biotechnology company (Mashhad, Iran). SinaSYBR Blue HS-qPCRMix, 2x (Cat No., MM2171) was provided by Sinaclone (Tehran, Iran). 5.5'Dithiobis(2-nitrobenzoic acid) DTNB (Cat No., D218200), Hyamine 1622 (Cat No., 121-54-0), and butyrylthiocholine iodide (Cat No., B3253) were purchased from Sigma Aldrich (Saint Louis, MO, USA). Dulbecco's modified Eagle's medium (DMEM) was purchased from Gibco (Grand Island, USA). Fetal bovine serum (FBS) and penicillin streptomycin were purchased from Hangzhou Sijiqing Biological Engineering Materials Co. Ltd (Hangzhou, China). The HEK-293 cell line was provided from Pasteur Institute (Tehran, Iran).

\section{Cloning Process}

\section{Overview of the Vector}

pCMV-BChE was used as the standard plasmid in the positive control group and the parent plasmid was in highcopy number plasmid pBudCE4.1, which has the SV40 ori and $\mathrm{Bleo}^{\mathrm{R}}$ gene (Figure 1).

\section{Plasmid Construction and Validation (Digestion and Ligation)}

Specific sense and antisense primers flanked by SalI and KpnI, ScaI and XhoI sequences, respectively, were 


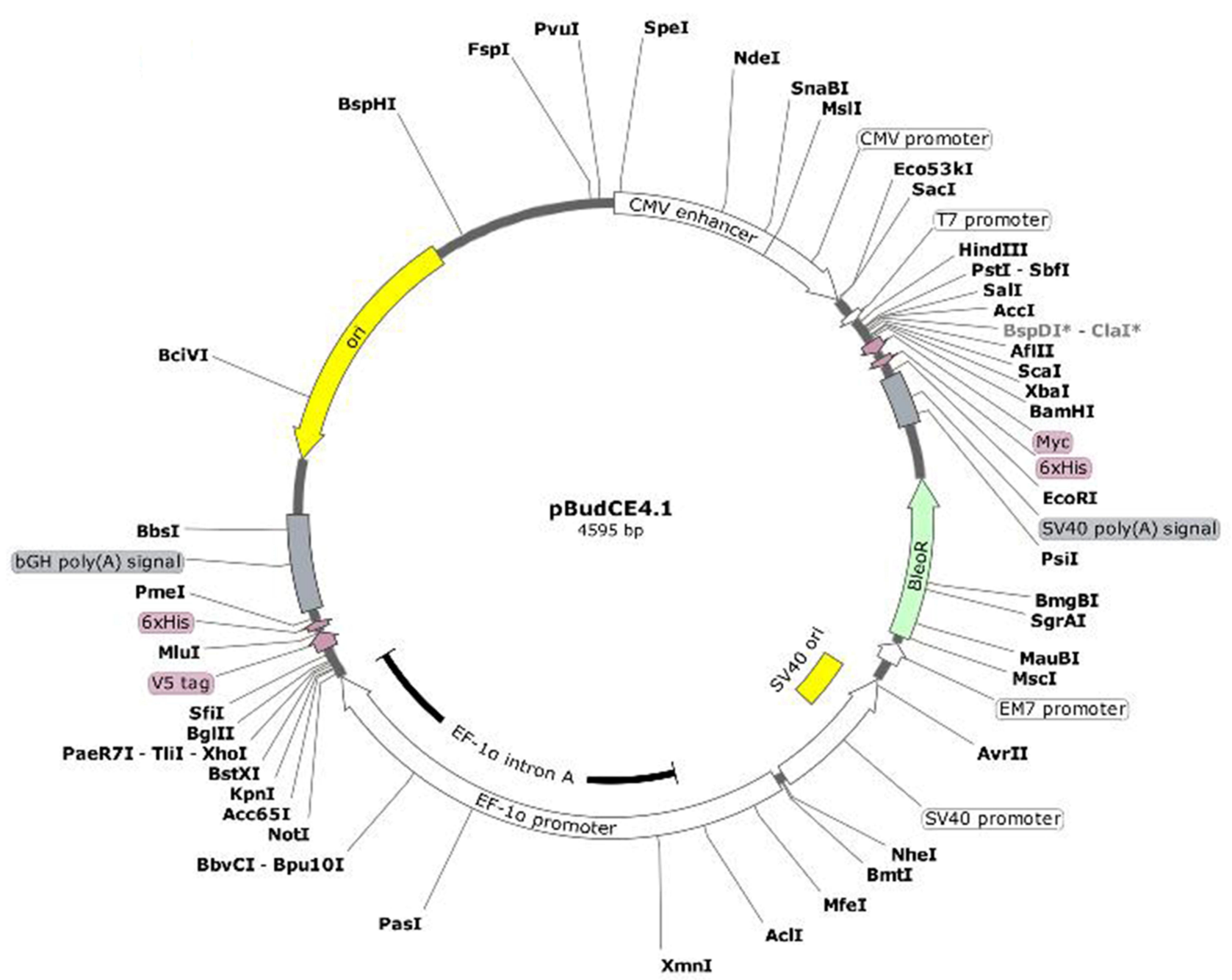

Figure I The schematic overview of the plasmid pBudCE4.I, containing two promoters of CMV and EF-I $\alpha$.

designed by NEBcutter ${ }^{\circledR}$ V2.0. Restriction enzyme sites were selected based on the pBudCE4.1 plasmid sequence (Figure 2).
cDNA of the BChE gene (gene ID: 590, $1809 \mathrm{bp}$ ) was synthesized by Takapozist company (Tehran, Iran). PCR amplification of the $B C h E$ gene was done using the

\section{$\mathbf{F}$}

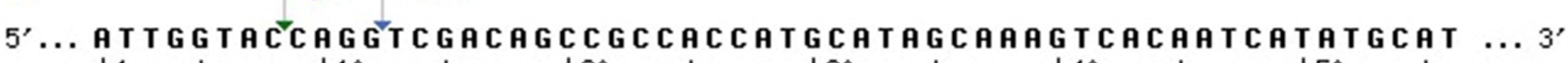
$r$ r $3^{\prime}$.... TAAC_CATGGTCCAGCTGTCGGCGGTGGTACGTATCGTTTCAGTGTTAGTATACGTA ... 5 ' KpnI

$\mathbf{R}$

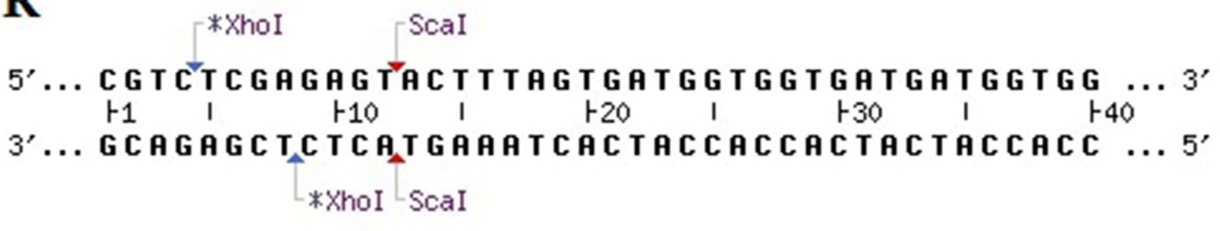

Figure 2 Restriction sites on the forward $(F)$ and reverse $(R) B C h E$ gene primers. 
mentioned primers according to the PCR program; $95^{\circ} \mathrm{C}$ for $7 \mathrm{~min}$, followed by 40 cycles of $95^{\circ} \mathrm{C}$ for $15 \mathrm{~s}, 65^{\circ} \mathrm{C}$ for $20 \mathrm{~s}$, and $72^{\circ} \mathrm{C}$ for $35 \mathrm{~s}$. PCR product and pBudCE4.1 plasmid were double digested by SalI, ScaI, and KpnI, XhoI enzymes, in separate tubes according to the manufacturer's protocol. Briefly, $1 \mu \mathrm{L}$ of each enzyme was added to $1 \mu \mathrm{L}$ DNA (concentration $1 \mu \mathrm{g}$ / $\mu \mathrm{L}), 2 \mu \mathrm{L}$ tango buffer ( $33 \mathrm{mM}$ Tris-acetate $(\mathrm{pH} 7.9$ at $37^{\circ} \mathrm{C}$ ), $10 \mathrm{mM}$ magnesium acetate, $66 \mathrm{mM}$ potassium acetate, $0.1 \mathrm{mg} / \mathrm{mL}$ BSA) and $15 \mu \mathrm{L}$ sterile water, the final volume reaching $20 \mu \mathrm{L}$. Tubes were incubated at $37^{\circ} \mathrm{C}$ for $1 \mathrm{~h}$ and inactivated at $65^{\circ} \mathrm{C}$ for $15 \mathrm{~min}$. The ligation process was done according to the manufacturer's protocol as follows: genes and plasmids which were digested by the same restriction enzymes were mixed and ligated by using the T4 ligation enzyme and buffer, and incubated at $22^{\circ} \mathrm{C}$ for $1 \mathrm{~h}$. The resulting products are two tubes containing two cloned plasmids, one with using SalI, ScaI and the other with KpnI, XhoI. Cloning validation was confirmed by digestion of each cloned plasmid with related digesting enzymes and the products were run on the agarose gel (Figure 3).

After determining that the gene was inserted into pBudCE4.1 plasmid, then pBudCE dual BChE vector was constructed as follows:

pBudCE4.1 plasmid was digested by SalI, ScaI, KpnI and $X h o I$ restriction enzymes: $1 \mu \mathrm{L}$ of plasmid, $1 \mu \mathrm{L}$ of each restriction enzyme $(4 \mu \mathrm{L}), 6 \mu \mathrm{L}$ tango buffer, and 19 $\mu \mathrm{L}$ sterile water, final volume $30 \mu \mathrm{L}$. Double-digested genes (gene digested by SalI, ScaI and KpnI, XhoI, separately) were added to the plasmid which is digested by all four enzymes afterward. The ligation process was done to construct the final form of the vector (pBudCE dual BChE) (Figure 4).

E. coli strain TOP10 competent cells prepared using the $\mathrm{CaCl}_{2}$ method and were transformed with the recombinant $\mathrm{pBudCE}$ dual $\mathrm{BChE}$ vector by the heat shock method. Transformed E. coli was cultivated at $37^{\circ} \mathrm{C}$ overnight in LB medium supplemented with Zeocin ${ }^{\mathrm{TM}}$ (50 $\mu \mathrm{g} /$ $\mathrm{mL}$ concentration). Plasmid extraction was done by GeneJET Plasmid Miniprep ${ }^{\circledR}$ Kit. The fresh LB/ Zeocin ${ }^{\mathrm{TM}}$ medium was inoculated with the transformed bacteria and incubated at $37^{\circ} \mathrm{C}$ for $16 \mathrm{~h}$. Bacterial cells were harvested by centrifugation at $6800 \times \mathrm{g}$ for $2 \mathrm{~min}$ and resuspended in lysis buffer $\left(50 \mathrm{mM} \mathrm{NaH} \mathrm{NO}_{4}\right.$, $300 \mathrm{mM} \mathrm{NaCl}, \mathrm{pH} 8.0$ ). Then, the plasmids were purified according to the protocol.

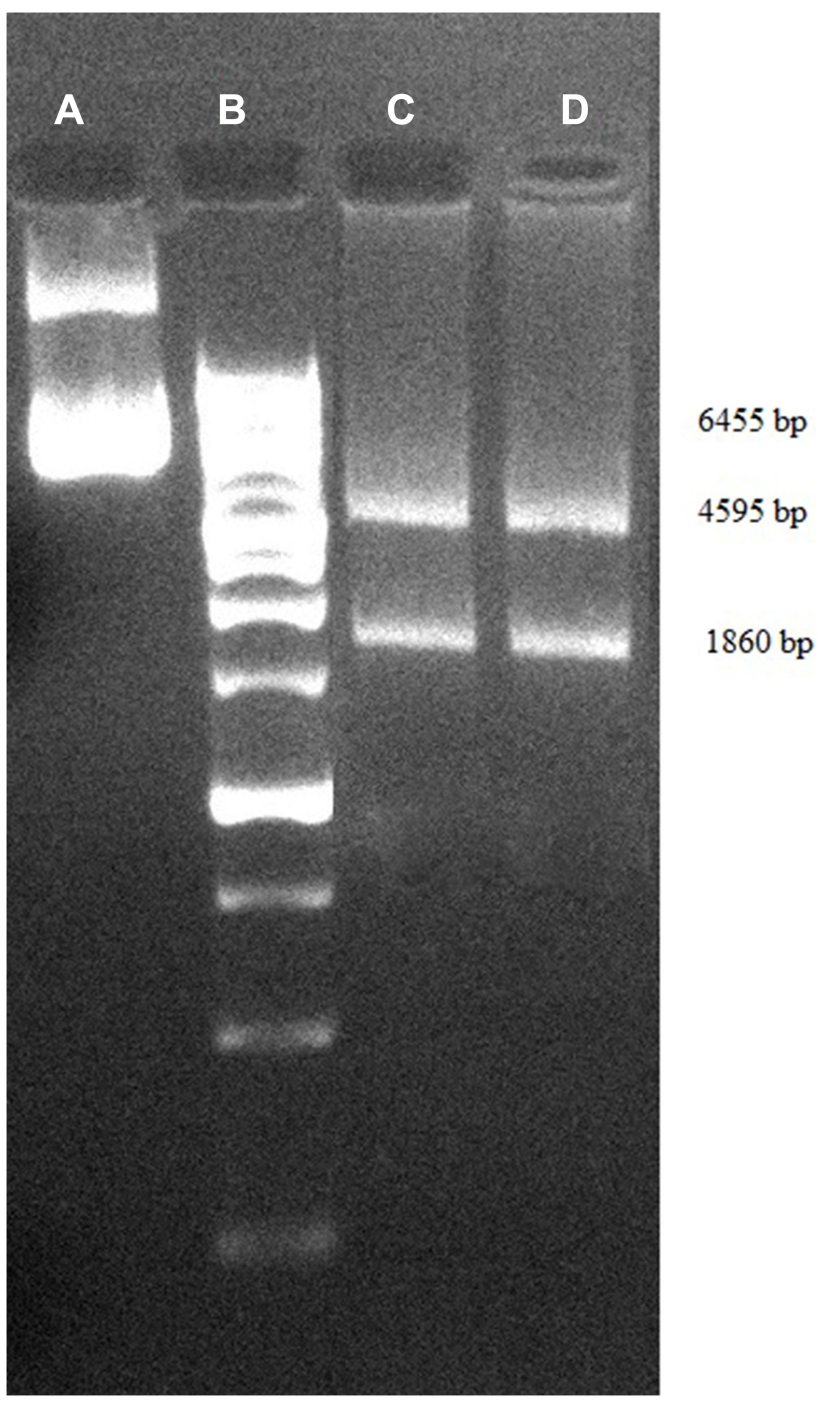

Figure 3 Cloning validation was confirmed by gel electrophoresis; (A) cloned plasmid, (B) 25kb ladder, (C) Sall, Scal digested products, (D) Kpnl, Xhol digested products.

\section{Preparation of Plasmids Decorated on Lipofectamine}

For each group (pCMV and pBudCE dual BChE), $14 \mu \mathrm{L}$ Lipofectamine $^{\mathrm{TM}}(2.5 \%)$ was added to $250 \mu \mathrm{L}$ serum-free medium in a microtube for liposomal formation, and $5 \mu \mathrm{g}$ of DNA plasmid (1\%) was diluted in $250 \mu \mathrm{L}$ serum-free medium in a separate microtube, simultaneously. The DNA suspension was gently added to the liposomal suspension afterward to the final volume of $500 \mu \mathrm{L}$. There were nine wells for each group; therefore, $50 \mu \mathrm{L}$ of the liposomal DNA was added to each well. The final DNA concentration was $0.5 \mu \mathrm{g} / \mathrm{well}$. 


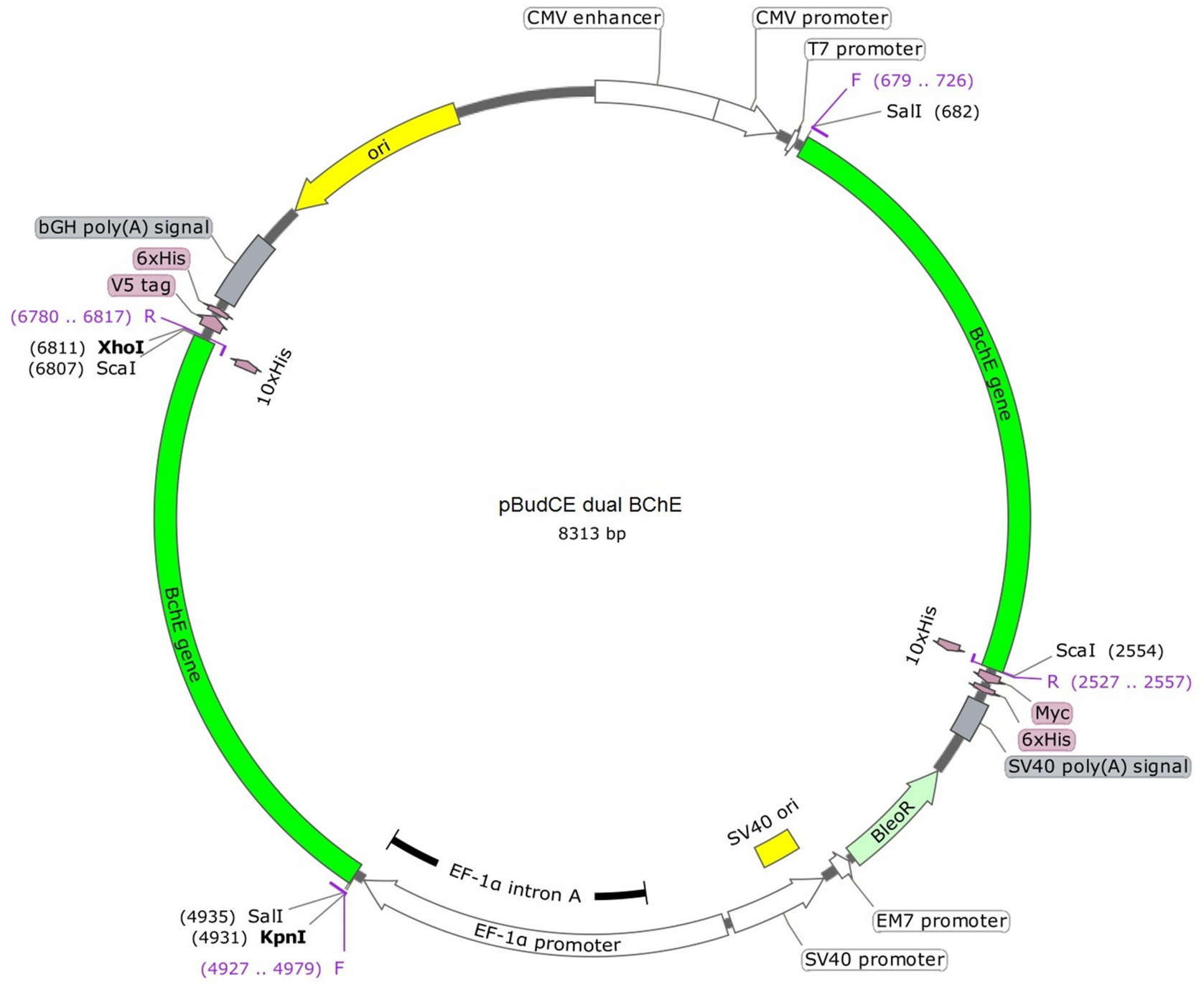

Figure 4 The final construction of the pBudCE dual BChE vector.

\section{Transfection and Cell Culture}

HEK-293 cells were grown in DMEM-F12 supplemented with $10 \% \mathrm{FBS}$ and were maintained in a humidified incubator with $5 \% \mathrm{CO}_{2}$ at $37^{\circ} \mathrm{C}$ to attain $90-95 \%$ confluency. Cells were trypsinized and re-suspended in the fresh serum-free $(2 \%$ FBS $)$ medium cell culture at a density of $2 \times 10^{5}$ cells $/ \mathrm{mL}$ one day before transfection. After incubating the cells for $24 \mathrm{~h}$, the medium was changed with $50 \mu \mathrm{L}$ of the prepared liposomal DNA suspension without antibiotics. The experimental groups were divided into transfected with pBudCE dual BChE (treatment), pCMV (positive control), and nontransfected cells (negative control) groups that have been followed for 3, 6, and 9 days. The culture medium of each group was collected for Ellman's method and the cells were lysed for RNA extraction and gene expression, separately.

\section{mRNA Extraction and Gene Expression}

Cells were lysed by adding $1 \mathrm{~mL}$ of RiboEx ${ }^{\mathrm{TM}}$ reagent following the manufacturer's instructions. Proteins were removed by phenol/chloroform extraction to obtain an $\mathrm{A}_{260}: \mathrm{A}_{280}$ ratio of $1.81 \pm 0.06$. The concentration and purity of isolated RNA were assessed by absorbance (A) readings on a UV spectrophotometer (Thermo Scientific ${ }^{\mathrm{TM}}$ NanoDrop 2000) at the wavelengths of 260 and $280 \mathrm{~nm}$. Synthesis of cDNA was carried out using a Parstous cDNA synthesis kit in $20 \mu \mathrm{L}$ of the final volume, according to the manufacturer's recommendations. For every first-strand cDNA synthesis, $0.1 \mu \mathrm{g}$ of total RNA was used, based on the following PCR program temperature as described below (Table 1).

Real-time PCR was performed using the SinaSYBER blue reaction mix without lowROX (SinaClon) in 
Table I RT-PCR Program for Synthesis of cDNA

\begin{tabular}{|l|l|l|}
\hline Steps & Temperature $\left({ }^{\circ} \mathbf{C}\right)$ & Time $(\mathbf{m i n})$ \\
\hline Primer annealing & 25 & 10 \\
Denaturation & 47 & 60 \\
Heat inactivation & 85 & 5 \\
\hline
\end{tabular}

a magnetic induction cycler (mic) real-time PCR system, using $1.5 \mu \mathrm{L}$ of each cDNA sample, and 10 pmol of each primer. The reaction was run as follows: $95^{\circ} \mathrm{C}$ for $7 \mathrm{~min}$, followed by 40 cycles of $95^{\circ} \mathrm{C}$ for $15 \mathrm{~s}, 65^{\circ} \mathrm{C}$ for $20 \mathrm{~s}$, and $72^{\circ} \mathrm{C}$ for $35 \mathrm{~s}$. The samples were electrophoresed to check the appearance of the PCR products. The $\mathrm{C}_{\mathrm{T}}$ (threshold cycle) values were analyzed using $2^{-\Delta \Delta C T}$ methods. ${ }^{22}$ The fold change in gene expression was normalized to an endogenous reference gene ( $\beta$-actin) using the following primers and expressed relative to the control (Table 2). micPCR software (Bio Molecular Systems, Australia) was used to evaluate the expression stability of the candidate reference gene.

\section{BChE Activity Assay}

BChE activity was determined using a modified Ellman's method. Briefly, $100 \mu \mathrm{L}$ of the supernatant was mixed with $3 \mathrm{~mL}$ of sterile $1 \times$ PBS. Then, $100 \mu \mathrm{L}$ of the resulting solution was transferred into a well of a 96-well culture plate. Butyrylcholinesterase isolated from human plasma (Sigma, USA) was used as a control to build the calibration curve. Then, $100 \mu \mathrm{L}$ of DTNB $(0.28 \mathrm{mmol})$ and 100 $\mu \mathrm{L}$ of butyrylcholine iodide $(3.2 \mathrm{mmol})$ were introduced into the wells containing the samples and controls and incubated at $37^{\circ} \mathrm{C}$ for $10 \mathrm{~min}$. The measurements were carried out at a wavelength of $412 \mathrm{~nm}^{23,24}$

\section{Stability Validation}

Transfected cells by pBudCE dual BChE vector were entered in the six freeze-thaw and subculture cycles consistently in order to analyze the stability of the cloning process. Transfection was done as explained before and after $24 \mathrm{~h}$ the cells were trypsinized and divided into two parts: frozen and cultured cells. The cultured cells were incubated under standard culture condition for six days, the medium was then collected as the first passage. The

Table 2 Primer Sequence of $\beta$-Actin Gene

\begin{tabular}{|l|l|}
\hline Gene & Primer Sequence \\
\hline$\beta$-actin & F: GGCTGTATTCCCCTCCATCG \\
& R: CCAGTTGGTAACAATGCCATGT \\
\hline
\end{tabular}

frozen cells were thawed the next day and again divided into the same two parts as mentioned above and continued the process until six passages. Finally, the collected medium of the six passages of the cells was analyzed with the modified Ellman's method.

\section{Freezing Procedure}

The cells were trypsinized and transferred to a sterile centrifuge tube, centrifuged at $1000 \mathrm{RPM}$ and $4^{\circ} \mathrm{C}$ for 3-5 min. The supernatant was removed and the cell pellet was re-suspended by adding $1 \mathrm{~mL}$ freezing medium $(10 \%$ dimethylsulfoxide, and $90 \% \mathrm{FBS}$ ) per vial. The procedure was continued by keeping the cryovial at $4^{\circ} \mathrm{C}$ for $1 \mathrm{~h}$, $-20^{\circ} \mathrm{C}$ for $2 \mathrm{~h}$, and $-80^{\circ} \mathrm{C}$ overnight. The next day cells were transferred to liquid $\mathrm{N}_{2}$ tank for $15 \mathrm{~min}$ and thawed afterward.

\section{Thawing Procedure}

Cryovial was removed from liquid $\mathrm{N}_{2}$ tank and hold in $37^{\circ} \mathrm{C}$ incubator until sides were thawed but the center remained frozen. Warm tissue culture medium $(0.5 \mathrm{~mL})$ was added to the vial and after pipetting gently, the cells were poured into a $3 \mathrm{~cm}$ plate. The medim was changed after $24 \mathrm{~h}$ and $1.5 \mathrm{~mL}$ fresh medium was added to the plate and maintained until 6 days.

\section{Statistical Analysis}

Data were presented as mean \pm SD while experiments were performed in triplicate. SPSS software 20 for Windows (SPSS Inc., Chicago) was utilized for statistical analysis. The univariate test was applied to determine the difference between the groups and Tukey's multiple range test was used to find the significant difference among means at the probability level of $\leq 0.05$. The nonparametric Kruskal-Wallis test was used to analyze stability data. Microsoft Office Excel 2010 was also used as appropriate software when required.

\section{Results \\ Cloning Validation}

E. coli bacteria were transfected with the recombinant pBudCE dual BChE vector. Colonies of the transformed bacteria were selected by the Zeocin ${ }^{\mathrm{TM}}(50 \mu \mathrm{g} / \mathrm{mL})$ supplemented liquid broth (LB) agar medium that confirmed the validity of the cloning and transformation process. Gel electrophoresis results of digestion of the recombinant vectors showed that each digested product had two bands, one for the gene of interest (1860 bp) and the other one for the remaining part of the plasmid (4595 bp) (specific sites: SalI, ScaI and KpnI, Xhol). 
The cloned plasmid had a $6455 \mathrm{bp}$ band and the control plasmid was 4595 bp (Figure 3).

\section{mRNA Extraction and Gene Expression}

Cells were lysed using RiboEx ${ }^{\mathrm{TM}}$ reagent and the purified RNA was used for cDNA synthesis. After PCR amplification of the gene with the cDNA, $B C h E$ gene expression was assessed using micPCR software (Bio Molecular Systems, Australia). Overall, results showed that the difference between groups was significant at $p \leq 0.05$ and the gene expression level in pBudCE dual BChE group was higher than in other groups in all days. Three days after inoculation the gene expression level in the negative control group was significantly lower than the $\mathrm{pBudCE}$ dual $\mathrm{BChE}$ and positive control (pCMV) groups. The gene expression level in pBudCE dual BChE was higher than the pCMV group, although the increase was not significant. The expression level fold changes were significantly different among all three groups at day six of inoculation, although on the ninth day, only the pBudCE dual $\mathrm{BChE}$ group showed a significant increase compared to the negative control group (Figure 5).

\section{Determining the BChE Enzyme Activity by Modified Ellman's Method}

The culture medium of each group was collected for Ellman's method. The results showed that the BChE activity in the pBudCE dual BChE group was significantly higher than that of the positive control (pCMV) and negative control groups. The difference of negative control, positive control, and pBudCE dual $\mathrm{BChE}$ groups was significant on third and sixth days of inoculation, while on the ninth day only the pBudCE dual $\mathrm{BChE}$ group was significantly different to the others. Although the enzyme activity in the pBudCE dual $\mathrm{BChE}$ group nonsignificantly decreased on the ninth day of inoculation compared with its level in the third and sixth days, it was higher than what was observed in other groups at any time point (Figure 6).

\section{Stability of the Transfected Cells}

The culture medium of each freeze-thaw cycle (6 cycles) of the transfected cells was harvested after six days in order to analyze the BChE enzyme activity by Ellman's method. The results showed that there was no significant difference among all passages of the cells $(p \leq 0.05)$. This means that the cloning process could be stable in the subculturing and freeze-thaw process (Table 3).

\section{Discussion}

Since the BChE enzyme is one of the main treatments in organophosphate toxin (OPT) exposures, and is introduced

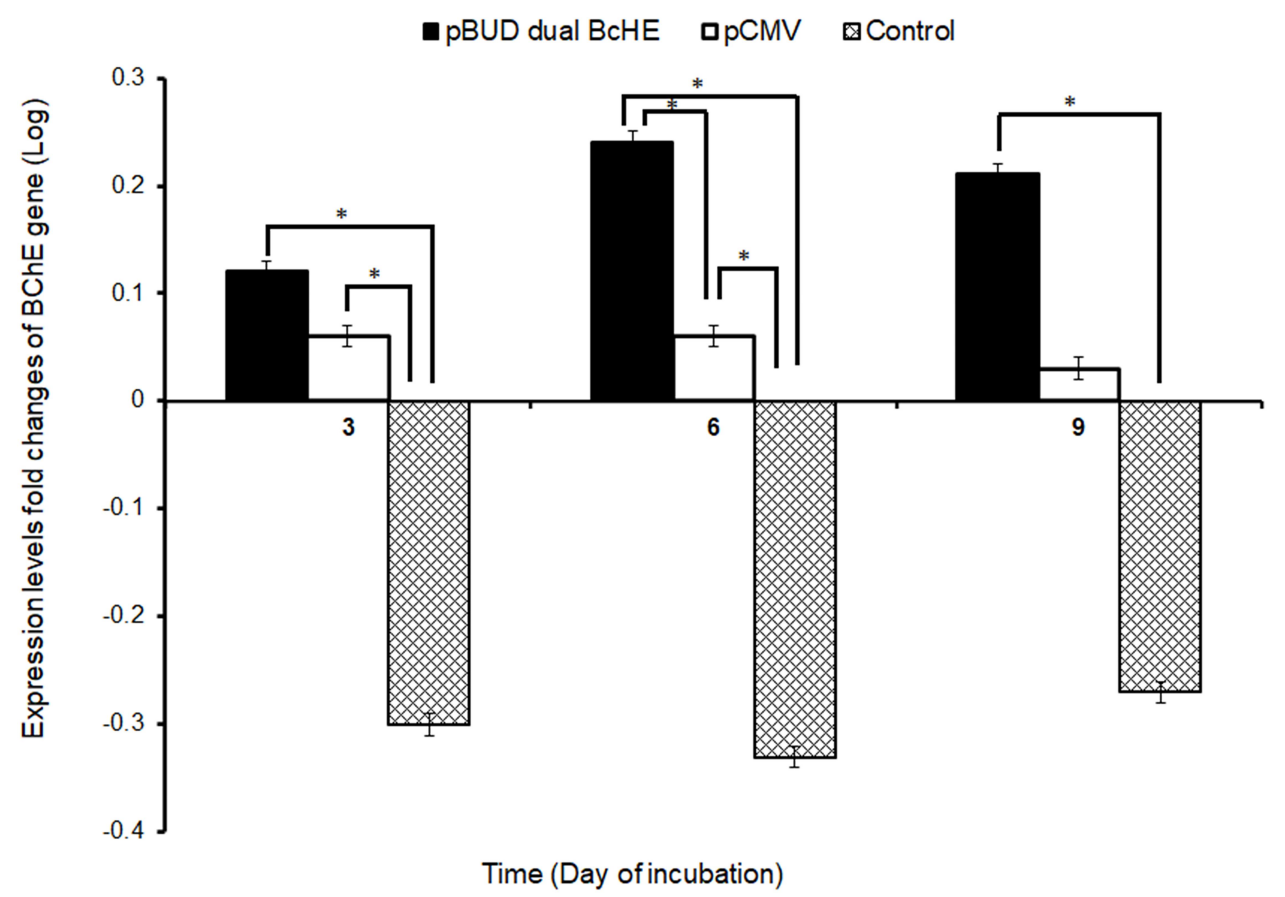

Figure 5 Expression levels of the $B C h E$ gene in the different experimental groups at different days of inoculation (mean \pm SD); $\left({ }^{*} p \leq 0.05\right)$. 


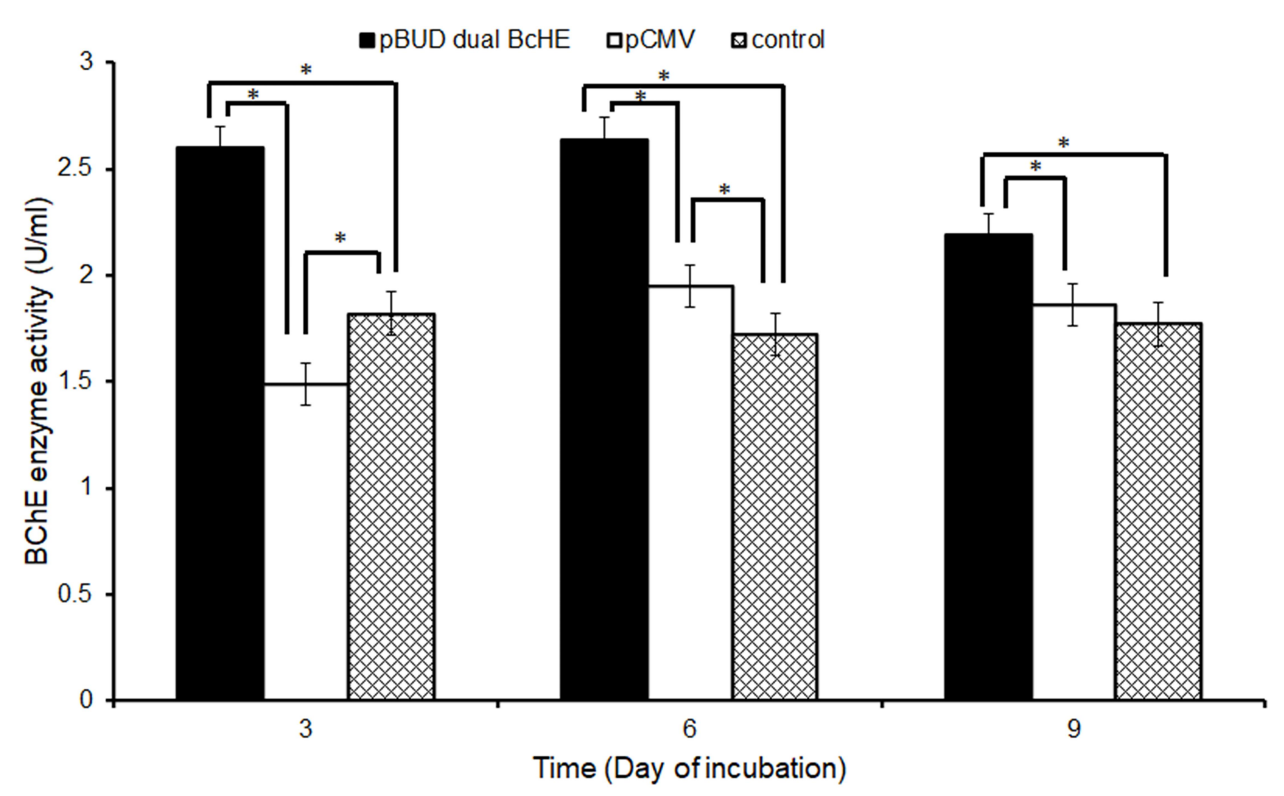

Figure 6 The BChE enzyme activity in different experimental groups during different days of inoculation. Data are presented as mean $\pm S D$ with dilution factor $=100$ folds; $(* p \leq 0.05)$.

as a drug in several therapeutic procedures, the aim of our study was to construct an efficient recombinant human $\mathrm{BChE}$ expression system to increase enzyme production. It is well documented that CMV and EF- $1 \alpha$ promoters are separately potent in recombinant protein production. However, we constructed a new dual-promoter vector to promote the plasmid's characteristics. The designed dual-promoter vector was capable of increasing the $B C h E$ gene expression level as well as the activity of the enzyme. Composition of a plasmid, choosing the best cell line and the most suitable vector are some of the essential factors that influence transgene expression magnitude, stability, and efficiency. Promoters have different ectopic gene expression patterns and potentials in various cell lines. EF-1 $\alpha$ and CMV are two well-studied and strong promoters in producing recombinant proteins in mammalian cell lines. They may affect the gene transcription, gene

Table 3 Stability Examination of the pBudCE Dual BChE Vector in the Transfected Cells During Six Freeze-Thaw Cycles and Passages

\begin{tabular}{|l|l|}
\hline Freeze-Thaw Cycle & Enzyme Activity $(\mathbf{U} / \mathbf{m L})$ \\
\hline I & $2.7 \pm 0.50$ \\
II & $3.0 \pm 0.30$ \\
III & $2.4 \pm 0.33$ \\
IV & $2.6 \pm 0.22$ \\
V & $2.2 \pm 0.21$ \\
VI & $3.0 \pm 0.31$ \\
\hline
\end{tabular}

expression, and protein synthesis in the cloning processes. ${ }^{25}$ CMV is mostly used in many biotechnological studies as a standard plasmid. ${ }^{21}$ Although CMV is a suitable promoter in terms of expression magnitude and stability, there are reports that with the extended cultivation periods, the productivity decreases by using this promoter. ${ }^{26}$ It has been reported that DNA methylation results in transcriptional silencing of the CMV promoter. By contrast, several strong promoters like EF-1 $\alpha$ have been exploited with the aim of long-term and potent expression of a gene. EF-1 $\alpha$ promoter tends to be more stable in long-term culture, even in the absence of selection pressure. It is constitutively active in a broad range of cell types and is able to increase the transgene expression, the transfection efficiency, the copy number of the vector, and the proportion of expression-positive clones. ${ }^{27}$ The EF-1a promoter is often active in cells in which viral promoters failed to express the controlled genes or are gradually silenced. ${ }^{28}$ In 2012 an expression system was designed comparing CMV and EF promoters separately to express the butyrylcholinesterase and the proline-rich region of the ColQ gene simultaneously by using two plasmids $^{24}$ or one plasmid ${ }^{29}$ in $\mathrm{CHO}$ cells. Their results demonstrated that the EF promoter produced higher levels of the active form of the $B C h E$ gene compared to the CMV promoter. Gene expression results in our study showed that the pCMV and pBUD dual BChE plasmids had comparable levels and they both could enhance the expression of the $B C h E$ gene significantly compared to the control; however, 
pBUD dual BChE plasmid showed higher levels. Although the pCMV vector increased the $B C h E$ gene expression, its level was significantly different from the control just at day three and six of inoculation. Meanwhile, the level of gene expression in new construction (pBUD dual BChE plasmid) was significantly higher at all three times of measurement which may be the effect of EF promoter. Sinae Kim and colleagues in 2007 evaluated the efficacy of promoters in the lentiviral gene delivery system in some mammalian ES cells to express EGFP. They used CMV and EF- $1 \alpha$ promoters, and, similarly to our results, they showed that the EF-1 $\alpha$ promoter had higher expression than that of the CMV promoter in all ES cells tested. ${ }^{30}$ Research investigating the importance of promoters for the generation of stable colon carcinoma cell lines showed that the level of stable clones generated with EF1- $\alpha$ promoter was much more than clones generated with CMV promoter. ${ }^{31}$ Another study in 2017 comparing various promoters to improve transgene expression, transgene stability, and copy number showed that the EF1- $\alpha$ promoter is a more potent promoter because compared to the other promoters including $\mathrm{CMV}^{27}$ which their results were comparable to ours that indicates; besides the effect of the presence of two copies of the gene in a single plasmid, EF- $1 \alpha$ promoter could play an important role in the protein production.

The HEK-293 cell line was selected as a candidate host in our study for $\mathrm{BChE}$ recombinant protein expression system. $\mathrm{CHO}$ cell lines are being used in many investigations as an effective transcription system while our results showed that HEK-293 could also be efficient as a cloning host. Epithelial origin and the biochemical machinery of the HEK-293 cell line make it capable to carry out most of the post-translational folding and processings which are required to generate functional and mature proteins from a wide range of both mammalian and non-mammalian nucleic acids. This cell line has many characteristics that make it appropriate for gene expression, eg, easy reproduction, high efficiency of transfection and protein production, and faithful translation and processing of proteins. ${ }^{20}$ Chilukuri et al investigated an AdenoVATOR system containing recombinant human butyrylcholinesterase in the Hek-293 cell line. Their results showed that most $\mathrm{rHu}$ $\mathrm{BChE}$ expressed in these cells was in tetrameric form by the co-expression of the proline-rich attachment domain. ${ }^{32}$

Many studies tried to produce recombinant $\mathrm{BChE}$ with several different systems, eg, transgenic animals, ${ }^{33}$ transgenic plants, ${ }^{25,34}$ and the $\mathrm{CHO}$ cell line. ${ }^{35}$ Although some of these studies reported some degrees of success, large quantity production of the
BChE enzyme is a major limitation so far. ${ }^{33,34,36,37}$ BChE enzyme activity was measured by a modified Ellman's method in the present study. Results showed BChE enzyme activity among pCMV, control and pBUD dual BChE groups was significantly different in all three periods of inoculation, and $\mathrm{pBUD}$ dual BChE group had the highest production level. Thus, the pBudCE dual $\mathrm{BChE}$ vector containing two copies of the $B C h E$ gene under the control of the EF- $1 \alpha$ and CMV promoters was the most promising construct. On the third day of inoculation, the activity level in the pCMV group was lower than the control group, while it increased in subsequent days. This may be due to the fact that the optimum function of this vector may begin from four days after transfection, as other studies mentioned. ${ }^{31}$ The most functional time for enzyme production with new construction is six days after transfection as the level of the enzyme activity decreased on day nine, although the difference was not significant. Nevertheless, the minimum level of the enzyme in the pBudCE dual BChE group at day nine was higher than the maximum level of the other groups.

The inoculation date did not significantly alter the results, although it is obvious that in both gene expression and enzyme activity, the highest level was observed six days after transfection. This result was comparable to the results of Jasmine et al that acclaimed the maximum expression level of the $B C h E$ gene is around day five of transfection. ${ }^{13}$ For this reason, this time (six days after inoculation) was selected for the stability test.

An inevitable factor in recombinant protein production is the durability and stability of the introduced method or the new construction. The production needs to be continued after some freeze-thaw or passages to become usable in a period of time. In order to examine the transient stability of the designed structure, the transfected cells with $\mathrm{pBudCE}$ dual $\mathrm{BChE}$ vector were entered into several freeze-thaw cycles. The transfected HEK-293 cells proved to be a stable $\mathrm{BChE}$ producer in six freeze-thaw cycles. Neither several generations nor the freeze-thaw process altered the BChE enzyme activity level, confirming the stability of the constructed vector. ${ }^{24}$

\section{Conclusion}

We can conclude that the designed construction containing EF-1 $\alpha$ and CMV promoters is a more efficient system than a single-promoter vector in protein and enzyme 
production. However, co-expression of the $B C h E$ gene and PRAD needs to be evaluated to achieve a high level of the tetrameric form of the enzyme. The HEK-293 cell line is an appropriate host in the cloning process that remains stable in several freeze-thaw and subculture procedures. Other cell lines than HEK-293 need to be evaluated in order to choose the most appropriate host for higher production of BChE.

\section{Acknowledgments}

This work was a part of the $\mathrm{PhD}$ thesis that was submitted under the ethics approval code: IR.KMU.REC.1398.294 in the Kerman University of Medical Sciences (KMU). M. Abolhassani from the department of clinical biochemistry at Afzalipour School of Medicine is acknowledged for his warm support.

\section{Funding}

This work was a part of the $\mathrm{PhD}$ thesis that was supported by the Kerman University of Medical Sciences (KMU) and submitted under the ethic approval code: IR.KMU. REC.1398.294.

\section{Disclosure}

The authors declare no conflict of interests for this work.

\section{References}

1. Çokuğraş AN. Butyrylcholinesterase: structure and physiological importance. Turk J Biochem. 2003;28(2):54-61.

2. Lockridge O, Bartels CF, Vaughan TA, Wong CK, Norton SE, Johnson LL. Complete amino acid sequence of human serum cholinesterase. J Biol Chem. 1987;262(2):549-557.

3. Østergaard D, Viby-Mogensen J, Hanel H, Skovgaard L. Half-life of plasma cholinesterase. Acta Anaesthesiol Scand. 1988;32(3):26 6-269. doi:10.1111/j.1399-6576.1988.tb02727.x

4. Duysen EG, Bartels CF, Lockridge O. Wild-type and A328W mutant human butyrylcholinesterase tetramers expressed in Chinese hamster ovary cells have a 16-hour half-life in the circulation and protect mice from cocaine toxicity. J Pharmacol Exp Ther. 2002;302(2):751-758. doi:10.1124/jpet.102.033746

5. Bosak A, Knežević A, Gazić Smilović I, Šinko G, Kovarik Z. Resorcinol, catechol-and saligenin-based bronchodilating $\beta 2$-agonists as inhibitors of human cholinesterase activity. J Enzyme Inhib Med Chem. 2017;32 (1):789-797. doi:10.1080/14756366.2017.1326109

6. Ashani Y. Prospective of human butyrylcholinesterase as a detoxifying antidote and potential regulator of controlled-release drugs. Drug Dev Res. 2000;50(3-4):298-308. doi:10.1002/1098-2299(200007/08)50:3/ 4<298::AID-DDR13>3.0.CO;2-X

7. Doctor BP, Saxena A. Bioscavengers for the protection of humans against organophosphate toxicity. Chem Biol Interact. 2005;157-158:167-171. doi:10.1016/j.cbi.2005.10.024

8. Eddleston M, Buckley NA, Eyer P, Dawson AH. Management of acute organophosphorus pesticide poisoning. Lancet. 2008;371 (9612):597-607. doi:10.1016/S0140-6736(07)61202-1
9. Gupta RD, Goldsmith M, Ashani Y, et al. Directed evolution of hydrolases for prevention of G-type nerve agent intoxication. Nat Chem Biol. 2011;7(2):120. doi:10.1038/nchembio.510

10. Cerasoli D, Griffiths E, Doctor B, et al. (07) in vitro and in vivo characterization of recombinant human butyrylcholinesterase (Protexia $^{\mathrm{TM}}$ ) as a potential nerve agent bioscavenger. Chem Biol Interact. 2005;157-158:362-365. doi:10.1016/j.cbi.2005.10. 052

11. Lenz DE, Yeung D, Smith JR, Sweeney RE, Lumley LA, Cerasoli DM. Stoichiometric and catalytic scavengers as protection against nerve agent toxicity: a mini review. Toxicology. 2007;233 (1-3):31-39. doi:10.1016/j.tox.2006.11.066

12. Ashani Y, Pistinner S. Estimation of the upper limit of human butyrylcholinesterase dose required for protection against organophosphates toxicity: a mathematically based toxicokinetic model. Toxicol Sci. 2004;77(2):358-367. doi:10.1093/toxsci/kfh012

13. Lockridge O, Schopfer LM, Winger G, Woods JH. Large scale purification of butyrylcholinesterase from human plasma suitable for injection into monkeys; a potential new therapeutic for protection against cocaine and nerve agent toxicity. $J$ Med Chem Biol Radiol Def. 2005;3:nihms5095.

14. Corbin JM, Hashimoto BI, Karuppanan K, et al. Semicontinuous bioreactor production of recombinant butyrylcholinesterase in transgenic rice cell suspension cultures. Front Plant Sci. 2016;7:412. doi: $10.3389 /$ fpls. 2016.00412

15. Griffiths AJ, Wessler SR, Lewontin RC, Gelbart WM, Suzuki DT, Miller JH. An Introduction to Genetic Analysis. Macmillan; 2005.

16. Khan S, Ullah MW, Siddique R, et al. Role of recombinant DNA technology to improve life. Int J Genomics. 2016;2016.

17. Masson P, Adkins S, Pham-Trong P, Lockridge O. Expression and refolding of functional human butyrylcholinesterase from E. Coli. In: Shafferman A., Velan B. (eds) Multidisciplinary Approaches to Cholinesterase Functions. Springer; 1992:49-52.

18. Brazzolotto X, Igert A, Guillon V, Santoni G, Nachon F. Bacterial expression of human butyrylcholinesterase as a tool for nerve agent bioscavengers development. Molecules. 2017;22(11):1828. doi:10.33 90/molecules 22111828

19. Rosano GL, Ceccarelli EA. Recombinant protein expression in Escherichia coli: advances and challenges. Front Microbiol. 2014;5:172. doi:10.3389/fmicb.2014.00172

20. Thomas P, Smart TG. HEK293 cell line: a vehicle for the expression of recombinant proteins. J Pharmacol Toxicol Methods. 2005;51 (3):187-200. doi:10.1016/j.vascn.2004.08.014

21. Qin JY, Zhang L, Clift KL, et al. Systematic comparison of constitutive promoters and the doxycycline-inducible promoter. PLoS One. 2010;5(5):e10611. doi:10.1371/journal.pone.0010611

22. Eslaminejad T, Noureddin Nematollahi-Mahani S, Ansari M. Glioblastoma targeted gene therapy based on pEGFP/p53-loaded superparamagnetic iron oxide nanoparticles. Curr Gene Ther. 2017;17(1):59-69. doi:10.2174/1566523217666170605115829

23. Ellman GL, Courtney KD, Andres Jr V, Featherstone RM. A new and rapid colorimetric determination of acetylcholinesterase activity. Biochem Pharmacol. 1961;7(2):88-95. doi:10.1016/0006-2952(61) 90145-9

24. Ilyushin D, Haertley O, Bobik T, et al. Recombinant human butyrylcholinesterase as a new-age bioscavenger drug: development of the expression system. Acta Naturae. 2013;5(1):16.

25. Schneider JD, Castilho A, Neumann L, et al. Expression of human butyrylcholinesterase with an engineered glycosylation profile resembling the plasma-derived orthologue. Biotechnol J. 2014a;9 (4):501-510. doi:10.1002/biot.201300229

26. He L, Winterrowd C, Kadura I, Frye C. Transgene copy number distribution profiles in recombinant $\mathrm{CHO}$ cell lines revealed by single cell analyses. Biotechnol Bioeng. 2012;109(7):1713-1722. doi:10.1002/ bit.24428 
27. Wang $\mathrm{X}, \mathrm{Xu} \mathrm{Z}$, Tian $\mathrm{Z}$, et al. The EF-1 $\alpha$ promoter maintains high-level transgene expression from episomal vectors in transfected CHO-K1 cells. J Cell Mol Med. 2017;21(11):3044-3054. doi:10.1111/jcmm.13216

28. Norrman K, Fischer Y, Bonnamy B, Sand FW, Ravassard P, Semb H. Quantitative comparison of constitutive promoters in human ES cells. PLoS One. 2010;5(8):e12413. doi:10.1371/journal.pone.0012413

29. Ilyushin DG, Smirnov IV, Belogurov AA, et al. Chemical polysialylation of human recombinant butyrylcholinesterase delivers a long-acting bioscavenger for nerve agents in vivo. Proc Natl Acad Sci. 2013;110(4):1243-1248. doi:10.1073/pnas.1211118110

30. Kim S, Kim GJ, Miyoshi H, et al. Efficiency of the elongation factor$1 \alpha$ promoter in mammalian embryonic stem cells using lentiviral gene delivery systems. Stem Cells Dev. 2007;16(4):537-546. doi: $10.1089 / \mathrm{scd} .2006 .0088$

31. Teschendorf C, Warrington JK, Siemann DW, Muzyczka N. Comparison of the EF-1 alpha and the CMV promoter for engineering stable tumor cell lines using recombinant adeno-associated virus. Anticancer Res. 2002;22(6A):3325-3330.

32. Chilukuri N, Parikh K, Sun W, et al. Polyethylene glycosylation prolongs the circulatory stability of recombinant human butyrylcholinesterase. Chem Biol Interact. 2005;157-158:115-121. doi:10.1016/j.cbi.2005.10.013
33. Huang Y-J, Huang Y, Baldassarre H, et al. Recombinant human butyrylcholinesterase from milk of transgenic animals to protect against organophosphate poisoning. Proc Natl Acad Sci. 2007;104 (34):13603-13608. doi:10.1073/pnas.0702756104

34. Geyer BC, Kannan L, Cherni I, Woods RR, Soreq H, Mor TS. Transgenic plants as a source for the bioscavenging enzyme, human butyrylcholinesterase. Plant Biotechnol J. 2010;8(8):873-886. doi:10.1111/j.1467-7652.2010.00515.x

35. Terekhov S, Smirnov I, Bobik T, et al. A novel expression cassette delivers efficient production of exclusively tetrameric human butyrylcholinesterase with improved pharmacokinetics for protection against organophosphate poisoning. Biochimie. 2015;118:51-59. doi:10.1016/j.biochi.2015.07.028

36. Brazzolotto X, Wandhammer M, Ronco C, et al. Human butyrylcholinesterase produced in insect cells: huprine-based affinity purification and crystal structure. FEBS J. 2012;279(16):2905-2916. doi:10.1111/j.1742-4658.2012.08672.x

37. Schneider JD, Marillonnet S, Castilho A, et al. Oligomerization status influences subcellular deposition and glycosylation of recombinant butyrylcholinesterase in $\mathrm{N}$ icotiana benthamiana. Plant Biotechnol J. 2014b;12(7):832-839. doi:10.1111/pbi.12184

\section{Publish your work in this journal}

Drug Design, Development and Therapy is an international, peerreviewed open-access journal that spans the spectrum of drug design and development through to clinical applications. Clinical outcomes, patient safety, and programs for the development and effective, safe, and sustained use of medicines are a feature of the journal, which has also been accepted for indexing on PubMed Central. The manuscript management system is completely online and includes a very quick and fair peer-review system, which is all easy to use. Visit http://www. dovepress.com/testimonials.php to read real quotes from published authors. 